\title{
Emergence of a Cosmological Constant in Anisotropic Fluid Cosmology
}

\author{
M. Cadoni ${ }^{a b *}$ A. P. Sanna ${ }^{a b \dagger}$ \\ ${ }^{a}$ Dipartimento di Fisica, Università di Cagliari \\ Cittadella Universitaria, 09042 Monserrato, Italy \\ ${ }^{b}$ I.N.F.N, Sezione di Cagliari, Cittadella Universitaria, 09042 Monserrato, Italy
}

December 16, 2020

\begin{abstract}
We investigate anisotropic fluid cosmology in a situation where the spacetime metric back-reacts in a local, time-dependent way to the presence of inhomogeneities. We derive exact solutions to the Einstein field equations describing Friedmann-Lemaitre-Robertson-Walker (FLRW) large scale cosmological evolution in the presence of local inhomogeneities and time-dependent back reaction. We use our derivation to tackle the cosmological constant problem. A cosmological constant emerges by averaging the back reaction term on spatial scales of the order of $100 \mathrm{Mpc}$, at which our universe begins to appear homogeneous and isotropic. We find that the order of magnitude of the "emerged" cosmological constant agrees with astrophysical observations and is related in a natural way to baryonic matter density. Thus, there is no coincidence problem in our framework.
\end{abstract}

\section{Introduction}

An anisotropic fluid is a two-fluid model, which can be used as an effective description of the sources of the gravitational field in several regimes of the gravitational interaction [1, 2, 3, 4, [5]. Originally proposed to describe local anisotropies in self-gravitating systems [1, 2, 5, recently it has gained renewed interest in the emergent and corpuscular [3, 6, 17, 8, 9, 10, 11] gravity context. In this framework it has been used to give a unified description of baryonic matter, dark energy and their possible interaction [3]. Specifically, at galactic scales, it can be used to explain the phenomenology commonly attributed to dark matter, in terms of a dark force originated by the reaction of dark energy (dark energy condensate in the corpuscular description) to the presence of baryonic matter. The corresponding additional acceleration component is generated by the radial pressure of the anisotropic fluid [3].

In a recent paper, the anisotropic fluid approach has been extended to the cosmological regime [4]. It has been shown that this type of fluid can be used to describe the transition from an inhomogeneous universe at short scales to a homogeneous one at large scales, during the dark energy-dominated epoch. The anisotropic stress of the fluid generates inhomogeneities and therefore could be responsible for structure formation at small scales. Using cosmological perturbation theory, a power spectrum for mass density distribution behaving as $1 / k^{4}$ was found, in good accordance with the observed two-point correlation function for matter distribution at small scales [4].

\footnotetext{
*E-mail: mariano.cadoni@ca.infn.it

†E-mail: asanna@dsf.unica.it
} 
The results of Ref. 4] were obtained in the decoupling limit, where the FLRW cosmological dynamics for the scale factor fully decouples from that describing inhomogeneities. This decoupling of degrees of freedom occurs both at the level of exact field equations and cosmological perturbation. The use of the decoupling limit is fully justified as long as one is only interested in structure formation at small scales in the dark energy-dominated era. On the other hand, one generically expects the presence of inhomogeneities to generate a time-dependent back reaction of the geometry.

Inclusion of the back reaction could be relevant for the cosmological model, i.e. the dynamics of the scale factor $a$. In particular, it could be in principle related to the appearance of a cosmological constant.

This is a quite interesting possibility because it could allow us to tackle the cosmological constant and the coincidence problems [10, 12, 13, 14, i.e to explain why the cosmological constant has exactly the value we observe and why in the present epoch it has the same order of magnitude of the density of baryonic matter. Moreover, showing the existence of a relation between back reaction of the spacetime geometry and the presence of a cosmological constant would corroborate the emergent gravity scenarios, where the latter is assumed, but not explained.

A related issue is the physical interpretation of the cosmological constant. It can be regarded as either a purely geometric term, when written on the l.h.s. of Einstein equations or as a source term, when written on the r.h.s. While the standard FLRW cosmology is based on the latter interpretation, the emergent gravity scenario naturally selects the former. This is consistent with the standard back reaction approach, which interprets the observational evidence of the accelerated expansion of the universe not as the presence of an exotic source, but as a non-linear response of the spacetime geometry to cosmological matter perturbations.

Following this line of thoughts, in this paper we derive, using an appropriate parametrization of the spacetime metric, exact solutions of Einstein's equations sourced by an anisotropic fluid. The model describes a FLRW universe at large scales, local inhomogeneities at small scales together with local, time-dependent back reaction of the geometry. We then apply these results to understand the origin of the cosmological constant. It emerges when we average the back reaction term on the spatial scales at which our universe appears homogeneous and isotropic. The resulting cosmological constant is related in a natural way to baryonic matter density - this solves the coincidence problem - and its order of magnitude is in agreement with astrophysical observations.

The structure of this paper is the following. In section 2 we briefly review the basics of anisotropic fluid cosmology. In section 3 we discuss the cosmological dynamics, including the back reaction of the metric to the presence of inhomogeneities. In section 4 we explore the emergence of a cosmological constant. Finally in section 5 we state our conclusions.

\section{Anisotropic fluid cosmology}

Anisotropic fluid cosmology is a generalization of the usual FLRW cosmology, which uses an anisotropic fluid to source cosmological evolution instead of the perfect fluid with barotropic equation of state. It is a two-fluid model, whose stress-energy tensor, after an appropriate redefinition of the velocities of the two barotropic fluids, can be written in terms of an anisotropic fluid with velocity $u_{\mu}$ and a spacelike vector $w_{\nu}$ (see, e.g. Ref. [15]). The stress-energy tensor for the fluid takes the form

$$
T_{\mu \nu}=\left(\rho+p_{\perp}\right) u_{\mu} u_{\nu}+p_{\perp} g_{\mu \nu}-\left(p_{\perp}-p_{\|}\right) w_{\mu} w_{\nu},
$$

where $u_{\mu}$ and $w_{\nu}$ satisfy $g^{\mu \nu} u_{\mu} u_{\nu}=-1, g^{\mu \nu} w_{\mu} w_{\nu}=1, u^{\mu} w_{\mu}=0$ and $\rho, p_{\|}, p_{\perp}$ are respectively the fluid density, pressures parallel and perpendicular to the spacelike vector $w_{\nu}$ [1, 2].

Taking $p_{\perp}=p_{\|} \equiv p$ and assuming a spatially homogeneous and isotropic spacetime metric, we get the usual FLRW cosmological model with $p, \rho$ and the scale factor of the metric depending on the cosmological time only. In this particular case, cosmological evolution is driven by a perfect fluid with barotropic equation of state $p=p(\rho)$, whereas the velocity field $u^{\nu}$ is free from rotation, shear and acceleration.

A non trivial anisotropic fluid is obtained by taking $p_{\perp} \neq p_{\|}$, generating an anisotropic stress. An important feature is that, although we have anisotropy in the stress-energy tensor, the spacetime metric may 
remain isotropic if homogeneity of the geometry is broken by allowing $p_{\perp}, p_{\|}$and $\rho$ to develop a dependence from the radial coordinate $r$. These features of the anisotropic fluid have been used (a) in Ref. 3 , to generate through $p_{\|}(r)$ a "dark force" at galactic level, which can explain the galactic phenomenology without assuming the existence of dark matter, and (b) in Ref. [4] in the cosmological framework, to describe structure formation at small scales in the late, dark-energy dominated universe. On the other hand, one should keep in mind that the anisotropic fluid cosmological model is unable to describe the early-time cosmology and the radiation/baryonic matter dominated eras, at least in the form given in Ref. [4. This is because any mass distribution is intrinsically unstable in the FLRW cosmology.

In the setup of Ref. [4, the spacetime metric is assumed to be isotropic but not homogeneous. It turns out that it is determined by two metric functions only - the conformal factor $a(t)$ and a metric function $f(r)$ which separately depend on the conformal time $t$ and the radial coordinate $r$ :

$$
d s^{2}=a^{2}(t)\left[-d t^{2}+f^{-1}(r) d r^{2}+r^{2} d \Omega^{2}\right] ; \quad d \Omega^{2}=d \theta^{2}+\sin ^{2} \theta d \phi^{2} .
$$

The scale factor $a(t)$ describes the cosmological dynamics, whereas $f(r)$ is determined by the inhomogeneities in the distribution of baryonic matter at small scales. A nice feature of the parametrization $(2)$ is that the dynamics for $a(t)$ and $f(r)$ fully decouples [4. The scale factor $a$ is determined by FLRW cosmological equations, whereas $f(r)$ is determined by the the Misner-Sharp mass $m_{B}(r)$ associated with the inhomogeneity density $\mathcal{E}(r)$ in the baryonic matter, $m_{B}(r)=4 \pi \int d r r^{2} \mathcal{E}(r)$, through the equation [4]:

$$
f=1-\frac{2 G m_{B}(r)}{r} .
$$

The inhomogeneities generate a contribution $\mathcal{P}(r)$ to the radial pressure $p_{\|}$given by [4:

$$
\mathcal{P}(r)=-\frac{m_{B}(r)}{4 \pi r^{3}}
$$

The decoupling between $a$ and $f$ is consequence of the parametrization of the metric (2), which neatly separates time dependence from radial dependence. This is natural and satisfactory as long as we are interested in describing cosmological dynamics at large scales and structure formation at small scales in a dark energy-dominated era. However, in a generic situation, we expect a time-dependent back reaction of the metric to the presence of local inhomogeneities. In this situation, the metric parametrization (2) is not satisfactory anymore and we expect the dynamics of $a$ to be coupled to that of $f$. This will be the topic of the next section.

\section{Inhomogeneous cosmological dynamics with back reaction on the metric}

In order to describe time-dependent back reaction of the metric to the presence of inhomogeneities in the context of anisotropic fluid cosmology, we need a generalization of the metric parametrization (2). We want to preserve spherical symmetry, we will therefore assume the following:

$$
d s^{2}=a^{2}(t)\left[-e^{\alpha(t, r)} d t^{2}+e^{\beta(t, r)} d r^{2}+r^{2} d \Omega^{2}\right]
$$

where $\alpha(t, r)$ and $\beta(t, r)$ are metric functions depending on both $t$ and $r$.

The resulting Einstein equations, sourced by an anisotropic fluid with stress-energy tensor given by Eq. (1), take the form:

$$
\begin{gathered}
3 \frac{\dot{a}^{2}}{a^{2}}+\frac{e^{\alpha-\beta}}{r^{2}}\left(-1+e^{\beta}+r \beta^{\prime}\right)+\frac{\dot{a}}{a} \dot{\beta}=8 \pi G e^{\alpha} a^{2} \rho \\
\frac{\dot{a}}{a} \alpha^{\prime}+\frac{\dot{\beta}}{r}=0 ;
\end{gathered}
$$




$$
\begin{gathered}
\frac{\dot{a}^{2}}{a^{2}} e^{\beta-\alpha}+\frac{1-e^{\beta}+r \alpha^{\prime}}{r^{2}}+e^{\beta-\alpha}\left(-2 \frac{\ddot{a}}{a}+\frac{\dot{a}}{a} \dot{\alpha}\right)=8 \pi G e^{\beta} a^{2} p_{\|} ; \\
\frac{e^{-(\alpha+\beta)}}{4 a^{2}} r\left\{4 e^{\beta} r \dot{a}^{2}+4 e^{\beta} r a \dot{a}(\dot{\alpha}-\dot{\beta})+a\left[-8 e^{\beta} r \ddot{a}+a\left[e^{\alpha} r \alpha^{\prime 2}-2 e^{\alpha} \beta^{\prime}-e^{\alpha} \alpha^{\prime}\left(-2+r \beta^{\prime}\right)+2 e^{\alpha} r \alpha^{\prime \prime}+\right.\right.\right. \\
\left.\left.\left.+e^{\beta} r\left(\dot{\alpha} \dot{\beta}-\dot{\beta}^{2}-2 \ddot{\beta}\right)\right]\right]\right\}=8 \pi G a^{2} r^{2} p_{\perp} .
\end{gathered}
$$

The dot and the prime denote derivatives with respect to $t$ and $r$, respectively.

Conservation of the stress-energy tensor gives two more equations:

$$
\begin{gathered}
\dot{\rho}+\frac{\dot{a}}{a}\left(3 \rho+p_{\|}+2 p_{\perp}\right)+\frac{\dot{\beta}}{2}\left(\rho+p_{\|}\right)=0, \\
p_{\|}^{\prime}+\frac{\alpha^{\prime}}{2}\left(\rho+p_{\|}\right)+\frac{2}{r}\left(p_{\|}-p_{\perp}\right)=0 .
\end{gathered}
$$

Eq. (7) can be immediately integrated to give

$$
\alpha=\mathcal{A}(t)-\frac{a}{\dot{a}} \int \dot{\beta} \frac{d r}{r}
$$

where $\mathcal{A}(t)$ is an arbitrary integration function.

We can now use Eq. (11) to express $p_{\perp}$ in terms of $p_{\|}$and $\rho$ :

$$
p_{\perp}=\frac{r}{2} p_{\|}^{\prime}+p_{\|}+\frac{\alpha^{\prime} r}{4}\left(\rho+p_{\|}\right) .
$$

Using this result, the field equations (6), (8) and (9) can be appropriately combined to give:

$$
\dot{\alpha} \dot{\beta}=0 .
$$

It follows that the field equations allow for two classes of solutions characterized either by $\dot{\beta}=0$ or by $\dot{\alpha}=0$. Let us discuss the two cases separately.

\subsection{The decoupling limit}

The case $\dot{\beta}=0$ corresponds to the decoupling of cosmological dynamics (the dynamics of the scale factor $a$ ) from that of inhomogeneities discussed in Sect. 2. In fact, in this case the metric function $e^{\beta}$ is a function of the radial coordinate $r$ only. Moreover, Eq. (12) implies $\alpha=\mathcal{A}(t)$ so that the metric function $e^{\alpha}$ can be completely absorbed by a redefinition of the conformal time $t$. We are left with only two degrees of freedom, the conformal factor of the metric $a$, which describes the cosmological dynamics, and the metric function $e^{\beta(r)}$, which describes inhomogeneities. Using the notation of Eq. (2) we have $e^{\beta(r)}=f^{-1}$, with $f$ given by Eq. (3).

In this situation we have exactly the case discussed in Sect. 2, where we have seen that the cosmological dynamics for $a$ is described by FLRW equations and decouples completely from that of inhomogeneities described by $e^{\beta(r)}=f^{-1}$. Notice that, owing to Eq. [12), the case $\dot{\beta}=0$ is completely equivalent to $\dot{\beta}=\dot{\alpha}=0$.

\subsection{Cosmological dynamics with back reaction of the metric}

The case $\dot{\alpha}=0$, when the metric function $e^{\alpha}$ depends only on $r$, describes the situation in which the metric responds to the presence of inhomogeneities by developing also time dependence of the metric function $e^{\beta}$. The cosmological degree of freedom $a$ is not decoupled from $\alpha$ and $\beta$ anymore. The dynamical equation for $a$ will therefore depend on the presence of the inhomogeneities (back reaction). 
Being $\alpha=\alpha(r)$ a function of the radial coordinate $r$ only, solving Eq. 12 for $\beta$ yields

$$
e^{-\beta}=g(r) a^{r \alpha^{\prime}(r)}
$$

where $g(r)$ is an integration function.

Using Eq. (7), the Einstein field equations and energy-momentum conservations take the form

$$
\begin{aligned}
& \frac{\dot{a}^{2}}{a^{2}}\left(3-r \alpha^{\prime}\right) e^{-\alpha}+\frac{1-e^{-\beta}+r \beta^{\prime} e^{-\beta}}{r^{2}}=8 \pi G a^{2} \rho \\
& \frac{e^{-\beta}+r e^{-\beta} \alpha^{\prime}-1}{r^{2}}+e^{-\alpha}\left(-2 \frac{\ddot{a}}{a}+\frac{\dot{a}^{2}}{a^{2}}\right)=8 \pi G a^{2} p_{\|} \\
& \dot{\rho}+\frac{\dot{a}}{a}\left(3 \rho+3 p_{\|}+r p_{\|}^{\prime}\right)=0
\end{aligned}
$$

together with Eq. 15 giving $\beta$ in terms of $\alpha$ and the arbitrary function $g(r)$. As usual in FLRW cosmology, the conservation equation $16 \mathrm{c}$ is not independent, but is a consequence of the field equations, so that we are left with a system of three independent equations for $\alpha(r), \beta(r, t), a(t), \rho(r, t), p_{\|}(r, t)$.

General, analytic solution of Eqs. 15, 16a, 16b are difficult to find. Following a method similar to that used in Ref. [4, we will look for solutions by separating the equations for the conformal factor $a$ from those for $\alpha$ and $\beta$. Although a full decoupling of these degrees of freedom is not possible in this case, one can still rewrite Eqs. 16a, 16b in terms of separate equations. This can be very useful when the time-scale of variation of $a$ is much larger than that of the function $\beta$.

Let us first define rescaled density $\hat{\rho}$ and pressure $\hat{p}_{\|}$for the fluid:

$$
\hat{\rho} \equiv \frac{3 e^{\alpha}}{3-r \alpha^{\prime}} \rho ; \quad \hat{p}_{\|} \equiv e^{\alpha} p_{\|}
$$

Rewritten in terms of these rescaled quantities, Eqs. 16a, 16b take the form:

$$
\begin{aligned}
& a^{2} \hat{\rho}=\frac{3}{8 \pi G}\left(\frac{\dot{a}}{a}\right)^{2}+\frac{3 e^{\alpha}}{8 \pi G r^{2}} \frac{\partial_{r}\left(r-r e^{-\beta}\right)}{3-r \alpha^{\prime}} ; \\
& a^{2} \hat{p}_{\|}=\frac{1}{8 \pi G}\left[\left(\frac{\dot{a}}{a}\right)^{2}-2 \frac{\ddot{a}}{a}\right]+\frac{e^{\alpha}}{8 \pi G r^{2}}\left(e^{-\beta}+r e^{-\beta} \alpha^{\prime}-1\right) .
\end{aligned}
$$

Let us now assume that $\hat{\rho}$ and $\hat{p}_{\|}$can be separated in (1) a homogeneous, time-dependent part, (2) a inhomogeneous time-independent part and $(3)$ a $(t, r)$-dependent interaction part:

$$
\begin{aligned}
& a^{2} \hat{\rho}(t, r) \equiv a^{2} \rho^{(1)}(t)+\frac{3 e^{\alpha}}{3-r \alpha^{\prime}}\left(\rho^{(2)}(r)+\rho^{(3)}(r, t)\right), \\
& a^{2} \hat{p}_{\|}(t, r) \equiv a^{2} p_{\|}^{(1)}(t)+e^{\alpha}\left(p_{\|}^{(2)}(r)+p_{\|}^{(3)}(r, t)\right) .
\end{aligned}
$$

The physical intuition behind the ansatz 19 is that the anisotropic fluid sourcing gravity allows for a separation between the purely time-dependent cosmological dynamics sourced by $\rho^{(1)}(t)$ and that of inhomogeneities sourced by $\rho^{(2)}(r)$, whereas $\rho^{(3)}(r, t)$ represents an interaction term (back reaction) whose form we expect to be constrained by the sources $\rho^{(1)}$ and $\rho^{(2)}$.

Using the ansatz $(19)$ we can separate Eqs. 18a, , (18b) into (1) a purely time-dependent part, which determines the scale factor $a$ and takes a FLRW form; $(2)$ a $(t, r)$ part, which determines the metric function $\beta$ in terms of the densities $\rho^{(2,3)}$, and (3) a relation determining the pressures $p_{\|}^{(2,3)}$.

More specifically we get from the purely time-dependent part

$$
\begin{aligned}
& a^{2} \rho^{(1)}(t)=\frac{3}{8 \pi G}\left(\frac{\dot{a}}{a}\right)^{2}, \\
& a^{2} p_{\|}^{(1)}(t)=\frac{1}{8 \pi G}\left[\left(\frac{\dot{a}}{a}\right)^{2}-2 \frac{\ddot{a}}{a}\right],
\end{aligned}
$$


which are the FLRW cosmological equations written in conformal frame $d s^{2}=a^{2}(t)\left(-d t^{2}+d r^{2}+r^{2} d \Omega^{2}\right)$ for the case of zero spatial curvature.

The metric function $e^{-\beta}$ can be determined from Eq. 18a and turns out to be:

$$
e^{-\beta}=1-\frac{2 G M(t, r)}{r}, \quad M(t, r) \equiv M^{(1)}(t)+M^{(2)}(r)+M^{(3)}(r, t) .
$$

Here the time-dependent mass term $M^{(1)}(t)$ is an integration function, whereas $M^{(2,3)}$ are the masses associated with inhomogeneities and the interaction, respectively:

$$
M^{(2)}(r)=4 \pi \int d r r^{2} \rho^{(2)}(r), \quad M^{(3)}(r, t)=4 \pi \int d r r^{2} \rho^{(3)}(r, t) .
$$

The other metric function $\alpha$ is then determined using Eq. 12 , giving:

$$
\alpha=\frac{1}{\ln a} \int \frac{d r}{r} \ln \left(\frac{1}{g}-\frac{2 G M}{g r}\right) .
$$

Eq. $18 \mathrm{~b}$ determines $\beta$ in terms of $p_{\|}^{(2,3)}$ and $\alpha$, which upon using Eqs. 21, 23 gives:

$$
p_{\|}^{(2)}=-\frac{M^{(2)}(r)}{4 \pi r^{3}}, \quad p_{\|}^{(3)}=-\frac{M^{(1)}(t)+M^{(3)}(r, t)}{4 \pi r^{3}}+\frac{1}{8 \pi G r^{2} \ln a}\left(1-\frac{2 G M}{r}\right) \ln \left(\frac{1}{g}-\frac{2 G M}{g r^{2}}\right) .
$$

Notice that the solutions $21, \sqrt{22}, p_{\|}^{(2)}$ and the first term in $p_{\|}^{(3)}$ of 24 have the same form of those appearing in Eqs. (3) and (4) in the decoupling limit. The mass $M(r, t)$ in Eq. (21) is a $t, r$-dependent Misner-Sharp mass. Moreover, the decoupling limit discussed in Sect. 2 is obtained in the limit $\alpha=$ const. In this limit we can easily see that the interaction term is switched off: $M^{(1)}=M^{(3)}=p_{\|}^{(3)}=0$ so that equations (21), 222 match exactly Eqs. (3) and (4).

It is important to stress that Eq. 23 implies a strong constraint on the form of the mass function $M(t, r)$. This is because the l.h.s of Eq. (23) is a function of the radial coordinate $r$ only, whereas in its r.h.s functions of both $t$ and $r$ are present. $M$ has to be chosen in such way to cancel the overall dependence on $t$. As anticipated, this constraint is largely expected from a physical point of view. The only completely free contributions to $19 \mathrm{a}$ are the spatially homogeneous $\rho^{(1)}$ and inhomogeneous $\rho^{(2)}$ densities. They are determined respectively by the cosmological evolution and by the physics of the early universe, for which our cosmological model cannot be used as stated in Sect. 2. Their interaction term $\rho^{(3)}$, on the other hand, is not completely free but is constrained by the form of $\rho^{(1)}$ and $\rho^{(2)}$. A way to encode information about early time cosmology in our context is to assume a phenomenological relation between $p_{\|}^{(2)}$ and $\rho^{(2)}$, which would play a role analogous to the equation of state for the fluid in the usual FLRW cosmology. In Ref. 4] such relation was inspired by galactic dynamics. With the same logic one could assume a relation between $p_{\|}^{(3)}$ and $\rho^{(3)}$, but we will not elaborate on this point in this paper.

Finally, once $p_{\|}$and $\rho$ are known, the perpendicular pressure of the fluid can be easily computed using Eq. (13).

\section{Emergence of the cosmological constant}

In the emergent gravity framework, geometry and gravity are seen as macroscopic features emerging out of some quantum mechanical microscopic theory. The phenomenology commonly attributed to dark matter could also be explained as an emergent phenomenon, a dark force originated from the response of dark energy to the presence of baryonic matter [8]. An interesting realization of this scenario has been given in the corpuscular gravity context [3, 6, 7, where an effective description of dark energy and baryonic matter in terms of an anisotropic fluid has been proposed. 
In this emergent gravity framework, dark energy is assumed to be present from the beginning and until now no attempt to explain its origin has been made. On the other hand, the results of Ref. 4, show that also matter distribution at small cosmological scales can be explained in anisotropic fluid cosmology as due to inhomogeneities triggered by the fluid anisotropy. The results of Ref. [4 have been derived in the regime of cosmological dynamics for the scale factor $a$ fully decoupled from that of the inhomogeneities. It is therefore tempting to look at the interaction term in Eqs. (16a), $(16 \mathrm{~b})$, which describes the reaction of the geometry to the presence of inhomogeneities, as the source for dark energy.

In order to keep the discussion as simple as possible, we will consider the simplest case of dark energy, namely that of a cosmological constant $\Lambda$. In presence of a $\Lambda$ term, Eqs. (20a) and (20b) become:

$$
\begin{aligned}
& a^{2} \rho(t)=\frac{3}{8 \pi G}\left(\frac{\dot{a}}{a}\right)^{2}-\frac{\Lambda}{8 \pi G} a^{2} \\
& a^{2} p(t)=\frac{1}{8 \pi G}\left[\left(\frac{\dot{a}}{a}\right)^{2}-2 \frac{\ddot{a}}{a}\right]+\frac{\Lambda}{8 \pi G} a^{2} .
\end{aligned}
$$

If we want to obtain these equations from Eqs. (18a), $18 \mathrm{~b}$ ) we have to first solve two main conceptual difficulties. First, the general solution we found in Sect. 3 gives interaction terms in Eqs. (16a), (16b), which are a complicate function of the scale factor $a$. Moreover, as we have seen in the previous section, Eq. 23. represents an highly non trivial constraint on the form of $\beta$. Second, these terms have not the form of a constant multiplying the scale factor $a^{2}$.

The first difficulty can be solved by looking at Eqs. (25a), $25 \mathrm{~b}$ as the leading order in the expansion of Eqs. 118a), 18b near the decoupling limit, i.e. $\alpha=$ constant (or equivalently $r \alpha^{\prime}=0$ ) and near the present evolution stage of our universe, i.e $a^{2}=1$. This second limit is also justified by the fact that, according to present observations [16], the cosmological constant starts dynamically dominating over matter in the very late universe, at $z \sim 0.6$.

For what concerns the second difficulty, we will simply assume that the cosmological term results from spatial averaging the interaction terms in Eqs. $18 \mathrm{a}, 1 \mathrm{~b}$ at scales $R \approx 100 \mathrm{Mpc}$ at which our universe appears to be homogeneous.

The use of spatially averaged quantities is well motivated in cosmology. The cosmological principle, i.e. the homogeneity and isotropy of the universe, is valid only in a statistical sense and at large scales, whereas our universe is highly non homogeneous at small scales. Moreover, due to the non-linear character of Einstein's equations, spatial averaging and time evolution do not commute, an aspect whose importance for cosmology was first investigated in [17] and further emphasized in [18. This implies that the field equations obtained from a smoothed metric (the FLRW equations in the standard approach) are different from those obtained from a general inhomogeneous metric and then averaged on some scale. In the latter case, new terms appear, which describe the back reaction of inhomogeneities to geometry [19, 20, 21. These terms could potentially be important in the evolution of the universe (see, e.g. Refs. [21, 22]), for instance they could trigger the observed accelerated expansion of the universe [23, 24, 25, 26, 27, 28, 29.

Expanding (15) near $a^{2}=1$ and keeping only the terms up to order $\left(a^{2}-1\right)$ we get:

$$
e^{-\beta} \approx g(r)\left[1+\frac{r \alpha^{\prime}}{2}\left(a^{2}-1\right)\right]
$$

Using this expression and the separation ansatz 19a into Eq. 18a and separately equating terms depending only on $r$ and those containing also a $t$-dependence, we can find both $g$ and $\alpha^{\prime}$ :

$$
g(r)=1-\frac{2 G\left(M^{(2)}(r)+M^{(3)}(r)\right)}{r}, \quad g(r) r^{2} \alpha^{\prime}=-4 G M^{(3)}(r)=-16 \pi G \int d r r^{2} \rho^{(3)}(r) .
$$

Notice that the compatibility constraint now requires $M^{(1)}(t)=0$ and $\rho^{(3)}$ to be a function of $r$ only. 
Using back Eq. 27] into Eqs. 18a, 18b, keeping only the leading terms in the $\alpha=$ constant expansion we get:

$$
\begin{aligned}
& a^{2} \hat{\rho}=\frac{3}{8 \pi G}\left(\frac{\dot{a}}{a}\right)^{2}+\rho^{(2)}(r)+a^{2} \rho^{(3)}(r) \\
& a^{2} \hat{p}_{\|}=\frac{1}{8 \pi G}\left[\left(\frac{\dot{a}}{a}\right)^{2}-2 \frac{\ddot{a}}{a}\right]-\frac{M^{(2)}+2 M^{(3)}}{4 \pi r^{3}}-a^{2} \frac{M^{(3)}}{4 \pi r^{3}} .
\end{aligned}
$$

Eqs. 28a), 28b have been derived by starting from small cosmological scales, at which the decoupling limit discussed in Ref. [4] works. At this scales, inhomogeneities determines the formation of structures encoded in the power spectrum for the density matter distribution [4]. We are here interested in the large scale cosmological dynamics. The simplest way to describe the transition to larger scales is to average Eqs. 28a , 28b over the spatial coordinate $r$ at the typical scales at which our universe appears to be homogeneous and isotropic, i.e. $R \sim 100 \mathrm{Mpc}$.

The averaging procedure produces in Eqs. (28a), 28b both constant terms and time-dependent terms. One can easily see that the constant terms on the l.h.s. of our equations cancel those on the r.h.s. Physically this cancellation means that the time-independent part of the inhomogeneities plays a role only at small scales, where it is responsible for structure formation (and for the emergence of a dark force at galactic scales [3, 7]), whereas it is irrelevant at large scales. Moreover, the cancellation of the constant terms stemming from averaging the $r$-dependent terms in Eqs. 28a and $28 \mathrm{~b}$ is consistent with the fact that the spatial curvature of our universe is zero [16. In fact, a non-vanishing constant term in Eqs. (28a) and (28b) would give a non-zero spatial curvature.

For what concerns the time-dependent part, on the l.h.s of Eqs. 28a, 28b we have the spatial averaging of $\hat{\rho}$, which contains the terms depending on $\rho^{(1)}$ and $\rho^{(3)}$ of Eqs. 19 and similarly for $\hat{p}_{\|}$. Spatial averaging of these quantities at large scales will produce the observed density $\rho(t) \equiv\langle\hat{\rho}\rangle_{r}$ and pressure $p(t) \equiv\left\langle\hat{p}_{\|}\right\rangle_{r}$ of matter appearing in the resulting FLRW cosmological equations.

After taking the average over the spatial coordinate $r$ of $28 \mathrm{a}, 2 \mathrm{28 \textrm {b }}$ ) we get therefore the FLRW cosmological equations:

$$
\begin{aligned}
& a^{2} \rho(t)=\frac{3}{8 \pi G}\left(\frac{\dot{a}}{a}\right)^{2}+a^{2}\left\langle\rho^{(3)}\right\rangle_{r} \\
& a^{2} p(t)=\frac{1}{8 \pi G}\left[\left(\frac{\dot{a}}{a}\right)^{2}-2 \frac{\ddot{a}}{a}\right]-a^{2}\left\langle\frac{M^{(3)}}{4 \pi r^{3}}\right\rangle_{r}
\end{aligned}
$$

In order to calculate the $\rho^{(3)}$-depending terms in the previous equations, we need to make some educated guess about the form of $\rho^{(3)}(r)$. As $\rho^{(3)}$ is the energy density of the back reaction of the metric on the presence of the inhomogeneities, characterized by $\rho^{(2)}$, these two have to be of the same order of magnitude, i.e. we have $\rho^{(3)} \sim \rho^{(2)}$. Moreover, it is natural to assume that $\rho^{(3)}$ gives rise to an attractive force. The fact that it has to behave as an inverse power of $r$ implies that $\rho^{(3)}$ has to be negative, as we will see in a while. The perturbative analysis of Ref. [4] has shown that $\rho^{(2)} \sim \kappa_{1} / r+\kappa / r^{2}$, where $\kappa, \kappa_{1}$ are some constants. In Ref. 44 it was also shown that $\kappa=0$ in the small scale regime. We are here considering the large scale regime, at the transition to an homogeneous and isotropic universe, in which inhomogeneities are expected to die out more rapidly than at small scales. We are therefore led to put $\kappa_{1}=0$, so that we have:

$$
\rho^{(3)} \sim-\frac{\kappa}{r^{2}}
$$

The behavior of $\rho^{(3)} \sim r^{-2}$ is consistent with the standard cosmological scenario and with the results of Ref. [4. The large scale, inflationary, matter power spectrum $P(k) \sim k$ (where $k$ represents the wave number), is modified, approximately at the equivalence epoch, by the transfer function $T^{2}(k)$, i.e. $P(k) \sim$ $k T^{2}(k)$. At small scales we have $P(k) \sim k^{-3}$ (or also $P(k) \sim k^{-4}$ if one works with matter correlation functions [4]). The bending scale is $L \sim 50 \Omega_{0}^{-1} h^{-2} \mathrm{Mpc} \sim 300 \mathrm{Mpc}$ [12]. $L$ is therefore a bit higher than 
the scale $R$ we are considering for our model. Therefore, the $\rho^{(3)} \sim r^{-2}$ behavior corresponds to the power spectrum $P^{(3)}(k) \sim \frac{1}{k^{2}}$, which is intermediate between the large scale behavior $P(k) \sim k$ at scales bigger than $300 \mathrm{Mpc}$ and the small scale one $P^{(2)}(k) \sim k^{-4}$.

Using Eq. 31 in Eq. $29,, 30$ we can easily see that the equation of state $\left\langle p_{\|}^{(3)}\right\rangle_{r}=-\left\langle\rho^{(3)}\right\rangle_{r}$ holds. The same equations take the form given by Eqs. (25a), (25b). Hence, they describe a FLRW universe with cosmological constant $\Lambda$ given by:

$$
\Lambda \sim-8 \pi G\left\langle\rho^{(3)}\right\rangle_{r} \sim 8 \pi G\left\langle\rho^{(2)}\right\rangle_{r}
$$

Hence, the cosmological constant term appears on the r.h.s. of Eqs. 29, , 30 as the spatial average of a term describing the back reaction of the geometry to the presence of inhomogeneities. The interpretation of the cosmological constant as a negative term, i.e. a negative back reaction energy, in the in r.h.s. of Eqs. (29) instead of a positive term in its l.h.s., i.e a positive energy density of some form of matter, is fully consistent with the emergent gravity paradigm. The cosmological constant is not a form of (exotic) matter, but a geometric term describing back reaction of the geometry to the presence of usual baryonic matter.

Let us now estimate the order of magnitude of the cosmological constant given by Eq. (32) and compare our result with its observed value. Let us first write Eq. 32 in terms of the cosmological parameter $\Omega_{\Lambda 0} \equiv \Lambda / 3 \mathcal{H}_{0}^{2}$ and of the critical density $\rho_{c}=3 \mathcal{H}_{0}^{2} / 8 \pi G \sim 2 \cdot 10^{-29} \mathrm{~g} \cdot \mathrm{cm}^{-3}$, where $\mathcal{H}_{0}$ is the Hubble parameter. We get:

$$
\Omega_{\Lambda 0} \sim \frac{\left\langle\rho^{(2)}\right\rangle_{r}}{\rho_{c}}
$$

The scale $R$ on which we compute spatial average of inhomogeneities is the transition scale at which our universe becomes homogeneous, this is about $R \sim 100 \mathrm{Mpc} . \rho^{(2)}(r)$ is the density profile of baryonic matter so that we have

$$
\left\langle\rho^{(2)}\right\rangle_{r}=\frac{3 M}{4 \pi R}
$$

where $M$ is the total baryonic mass inside a sphere of radius $R$. For $R \sim 100 \mathrm{Mpc}$, we have $M \sim 10^{17} M_{\odot}$ and we get from Eq. (34) $\Omega_{\Lambda 0} \sim 10^{-1}$, which gives the correct order of magnitude of the observed cosmological constant [16]. Note that there is a strong dependence on the scale of averaging of the radial coordinate, namely our estimate agrees with observations only if we average at scales $\sim 100$ Mpc. The value of Eq. (33) changes drastically when changing the scale at which the average is performed. The observed order of magnitude of the cosmological constant is only obtained if the averaging scale is given by the transition scale at which our universe begins to appear homogeneous and isotropic.

Let us conclude this section with a final remark. Our model explains the emergence of the cosmological constant as a reaction of the geometry to inhomogeneities in the baryonic matter density distribution. Therefore, there is no coincidence problem [12, 13, [14. The fact that the energy densities associated to matter and $\Lambda$ are of the same order of magnitude at the present epoch is not a coincidence, but it is linked to the intrinsic nature of the emerged cosmological constant. Thus, in our model the origin of the accelerated expansion of the universe can be traced back to structure formation. Conversely, the coincidence problem represents a fierce challenge to the current standard cosmological model, since it requires extremely fine-tuned initial conditions in the early universe.

\section{Conclusions}

Building on previous work about anisotropic fluid cosmology [4], in this paper we have found exact solutions of Einstein's field equations sourced by an anisotropic fluid, which describe FLRW cosmological evolution at large scales, local inhomogeneities at small scales and a local, time-dependent back reaction of the geometry to the presence of the latter. These solutions generalize those found in Ref. 4 to the case in which the 
FLRW cosmological evolution of the scale factor does not decouple completely from local inhomogeneities. They could find applications as an alternative approach to the use of perturbations in late-time cosmology. In fact, in the usual setup of cosmological perturbation, the background metric is held fixed, whereas our approach allows to compute the back reaction of the metric.

A simple way to compute the effect of back reaction on the cosmological dynamics is to perform spatial averaging of the inhomogeneous terms. This is a quite conceptually involved point, because it reverses the usual cosmological paradigm, which first assumes homogeneity and isotropy and then uses perturbation theory in a homogenous and isotropic background. It is known that, owing to the non-linear character of Einstein's equations, spatial averaging and time evolution do not commute [17, 18. This implies that the field equations obtained from a general inhomogeneous metric averaged on some scale contain additional terms, whose effect could potentially be important in the evolution of the universe. For instance, they could trigger the observed accelerated expansion of the universe [23, 24, 25, 26, 27, 28, 29].

As an important application of this approach, we have considered the emergence of a cosmological constant $\Lambda$ as the result of spatial averaging the back reaction terms produced by local, time-dependent inhomogeneities in our anisotropic fluid cosmology. Specifically, we were able to reproduce the order of magnitude of the observed cosmological constant by averaging on scales of order $100 \mathrm{Mpc}$, at which our universe begins to appear homogeneous and isotropic.

Our solution of the cosmological constant problem is fully consistent with the emergent gravity paradigm. In fact, $\Lambda$ is described not as a form of (exotic) matter, but as a geometric term produced by the back reaction of the geometry to the presence of inhomogeneities in the baryonic matter distribution. This means that the accelerated expansion of our universe can be traced back to structure formation at scales smaller than those of the transition to homogeneity and isotropy. Since $\Lambda$ is determined by the spatial average of baryonic matter, the proposed solution to the cosmological constant problem has also the advantage of solving the coincidence problem at the same time. Being based on rough estimates of the parameters entering in the model (the mass $M$ and the averaging scale $R$ ), at least at the present stage of development, our model has not the predictive power to determine the exact ratio between baryonic matter and the cosmological constant, but only orders of magnitude.

Let us conclude with some general remarks about the use of anisotropic fluids in cosmology. They are very useful to describe several regimes of gravity: (1) at galactic scales, they produce an additional component of the radial acceleration [3]; (2) at small scales in late-time cosmology, they can explain structure formation [4]; (3) at large scale in late-time cosmology, they may explain the emergence of a cosmological constant, as shown in the present paper. Their use in cosmology, however, has a strong limitation: they cannot be used to describe early-time cosmology and the radiation/baryonic matter dominated eras. Mass distribution is intrinsically unstable in FLRW cosmology. So, any inhomogeneous cosmological model can describe the late-time epoch of the universe, cosmic structures at small scales and the back reaction of geometry to the presence of the latter, but not the early-time one nor the evolution of perturbations stemming from it.

This issue is strongly related with the fact that we use inhomogeneities to generate local anisotropic stresses -hence a non trivial anisotropic fluid. It may be possible that an alternative way to generate a non trivial anisotropic fluid does exist, for instance not using inhomogeneities but a different reparametrization of the original two-fluid description. Such an alternative description could open the way for using anisotropic fluids also in early-time cosmology.

\section{References}

[1] M. Cosenza, L. Herrera, M. Esculpi, and L. Witten (1981), "Some models of anisotropic spheres in general relativity", Journal of Mathematical Physics, 22(1), 118-125.

[2] L. Herrera, and N. O. Santos (1997), "Local anisotropy in self-gravitating systems", Physics Reports, 286(2), 53-130.

[3] M. Cadoni, R. Casadio, A. Giusti, W. Mueck, and M. Tuveri, "Effective Fluid Description of the Dark Universe," Phys. Lett. B776 (2018) 242-248, arXiv:1707.09945 [gr-qc] 
[4] M. Cadoni, A. P. Sanna and M. Tuveri, "Anisotropic fluid cosmology: An alternative to dark matter?," \begin{tabular}{|l|l|}
\hline Phys. Rev. D 102 (2020) no.2, 023514, arXiv:2002.06988 [gr-qc]. \\
\hline
\end{tabular}

[5] L. Herrera, "Stability of the isotropic pressure condition," Phys. Rev. D 101 (2020) no.10, 104024 arXiv:2005.06358 [gr-qc]

[6] M. Cadoni, R. Casadio, A. Giusti and M. Tuveri, "Emergence of a Dark Force in Corpuscular Gravity," Phys. Rev. D 97 (2018) no.4, 044047, arXiv: 1801.10374v1 [gr-qc].

[7] M. Cadoni and M. Tuveri, "Galactic dynamics and long-range quantum gravity," Phys. Rev. D 100, no. 2, 024029 (2019), arXiv:1904.11835 v2 [gr-qc].

[8] E. P. Verlinde, "Emergent Gravity and the Dark Universe," SciPost Phys. 2 no. 3, (2017) 016 , arXiv:1611.02269 [hep-th].

[9] G. Dvali and C. Gomez, "Black Hole's Quantum N-Portrait," Fortsch. Phys. 61 (2013), 742-767, arXiv:1112.3359 [hep-th].

[10] R. Casadio and A. Giusti, "Bootstrapped Newtonian cosmology and the cosmological constant problem," arXiv:2009.10667 [gr-qc]

[11] M. Cadoni, M. Tuveri and A. P. Sanna, "Long-Range Quantum Gravity," Symmetry 12 (2020) no.9, 1396, arXiv:2006.16652 [gr-qc]

[12] P. J. E. Peebles and B. Ratra, "The Cosmological Constant and Dark Energy," Rev. Mod. Phys. 75 (2003) 559, arXiv: astro-ph/0207347v2.

[13] H. E. S. Velten, R. F. vom Marttens and W. Zimdahl, "Aspects of the cosmological "coincidence problem"," Eur. Phys. J. C 74 (2014) no.11, 3160, arXiv:1410.2509 [astro-ph.C0].

[14] E. J. Copeland, M. Sami and S. Tsujikawa, "Dynamics of dark energy," Int. J. Mod. Phys. D 15 (2006), 1753-1936, arXiv:hep-th/0603057 [hep-th].

[15] S. S. Bayin, "Anisotropic fluids and cosmology," Astrophys. J. 303 (1986), 101-110.

[16] N. Aghanim, Y. Akrami, M. Ashdown, J. Aumont, C. Baccigalupi, M. Ballardini, ... and R. Battye (2018), "Planck 2018 results. VI. Cosmological parameters", arXiv:1807.06209v2 [astro-ph.C0].

[17] M. F. Shirokov and I. Z. Fisher, "Isotropic Space with Discrete Gravitational-Field Sources. On the Theory of a Nonhomogeneous Isotropic Universe," General Relativity and Gravitation 30 (1998), 1411-1427.

[18] G. F. R. Ellis, "Relativistic Cosmology: Its Nature, Aims and Problems," Fundam. Theor. Phys. 9 (1984), 215-288.

[19] T. Buchert, "On average properties of inhomogeneous fluids in general relativity. 1. Dust cosmologies," Gen. Rel. Grav. 32 (2000), 105-125, arXiv:gr-qc/9906015 [gr-qc]

[20] T. Buchert, "On average properties of inhomogeneous fluids in general relativity: Perfect fluid cosmologies," Gen. Rel. Grav. 33 (2001), 1381-1405, arXiv:gr-qc/0102049 [gr-qc].

[21] T. Buchert and S. Räsänen, "Backreaction in late-time cosmology," Ann. Rev. Nucl. Part. Sci. 62 (2012), 57-79, arXiv:1112.5335 [astro-ph.C0].

[22] G. F. R. Ellis, "Inhomogeneity effects in Cosmology," Class. Quant. Grav. 28 (2011), 164001 , arXiv: 1103.2335 [astro-ph.CO]. 
[23] E. Barausse, S. Matarrese and A. Riotto, "The Effect of inhomogeneities on the luminosity distanceredshift relation: Is dark energy necessary in a perturbed Universe?," Phys. Rev. D 71 (2005), 063537 arXiv:astro-ph/0501152 [astro-ph]

[24] E. W. Kolb, S. Matarrese and A. Riotto, "On cosmic acceleration without dark energy," New J. Phys. 8 (2006), 322, arXiv:astro-ph/0506534 [astro-ph].

[25] T. Buchert, "Dark Energy from Structure: A Status Report," Gen. Rel. Grav. 40 (2008), 467-527, arXiv:0707.2153v3 [gr-qc].

[26] M. N. Celerier, "The accelerated expansion of the Universe challenged by an effect of the inhomogeneities. A review," arXiv:astro-ph/0702416 [astro-ph].

[27] S. Rasanen, "Dark energy from backreaction," JCAP 02 (2004), 003 arXiv:astro-ph/0311257 [astro-ph].

[28] S. Rasanen, "Accelerated expansion from structure formation," JCAP $\mathbf{1 1}$ (2006), 003 arXiv:astro-ph/0607626 [astro-ph],

[29] T. Buchert and M. Carfora, "Cosmological parameters are dressed," Phys. Rev. Lett. 90 (2003), 031101. arXiv:gr-qc/0210045 [gr-qc]. 